\title{
Seismic wave attenuation beneath the Australasian region
}

\author{
B. L. N. KENNETT* AND A. ABDULLAH
}

Research School of Earth Sciences, The Australian National University, Canberra, ACT 0200, Australia

\begin{abstract}
The attenuation of seismic waves is strongly influenced by temperature and rheological changes, and so provides an important supplement to information from seismic wavespeeds. Differential attenuation measurements are made for seismic waves refracted through the mantle beneath the Australasian region and are then used to construct 3D images of the attenuation of shear and compressional waves. The differential attenuation between different seismic phases is estimated using a spectral ratio method in the frequency band $0.25-1.0 \mathrm{~Hz}$ using a multi-taper method. Over this band the attenuation is nearly independent of frequency so that the logarithmic spectral ratio is a linear function of frequency. Differential measurements are made either between $\mathrm{P}$ and S phases on the same record or between stations for P and S waves separately. Images of seismic attenuation for the Australasian region are produced using a tomographic inversion, with the fast marching method employed to trace ray paths in an initial 3-D model derived from surface wave tomography. There is a deep seated horizontal contrast between central Australia and the eastern seaboard. The crustal and lithospheric mantle beneath the Archean and Proterozoic rocks in the west and in the middle of the continent have low seismic attenuation, whereas the Phanerozoic material in the east is more attenuative. Regions with recent volcanism, most likely associated with hot spots such as near Bass Strait and the Coral Sea, display high seismic attenuation anomalies. There is a strong contrast in attenuation between the relatively low loss lithosphere and the high loss asthenosphere beneath with a change by a factor of 6-10. Anisotropy in attenuation is slight even though some would be expected from the radial anisotropy seen in shear wavespeed in the lithosphere.
\end{abstract}

KEY WORDS: seismic waves, attenuation, tomography, Australian continent, lithosphere, asthenosphere.

\section{INTRODUCTION}

Over the last two decades, a wide range of studies have been used to understand the 3-D structure in the mantle beneath the Australian continent by exploiting different aspects of seismograms. Many different methods have been employed (see e.g. Kennett 2003; Fichtner et al. 2010) with most attention directed to understanding the distribution of seismic wavespeeds. Most work has been carried out using the techniques of seismic tomography, either exploiting the seismic waveforms for the large amplitude surface waves that travel nearly horizontally, or using the travel times of body waves along thousands of propagation paths in which case, the sampling is closer to vertical.

Knowledge of the pattern of seismic attenuation in the Australian region is still limited. Seismic attenuation is important because it is particularly sensitive to temperature and rheological processes (see e.g. Jackson 2000 ), with a rapid increase in attenuation as the solidus is approached. Studies on seismic attenuation of the Australasian region were initiated by Gudmundsson et al. (1994) who presented an attenuation profile of the upper mantle beneath the north of the continent. This work indicated the presence of a zone of strong attenuation below the lithosphere under northern Australia but with a limited number of events, was not able to localise the behaviour. Subsequently Cheng (2000) studied Australian attenuation structure using the spectral ratio between $\mathrm{P}$ and $\mathrm{S}$ phases for nearly 2000 three-component seismograms for paths refracted back from the upper mantle recorded between 1993 and 1996. The differential attenuation data were organised into azimuthal corridors across the continent and then inverted for 1-D attenuation structure using the Neighbourhood Algorithm (Sambridge 1999). A quasi 3-D attenuation model at fixed frequency was then constructed by combining the set of 1-D attenuation profiles weighted by ray density. The results clearly delineate major contrasts in seismic attenuation between the cratonic structures in the centre and west of Australia and the younger eastern zone where attenuation is much stronger.

The continuing sequence of deployments of portable broadband seismic stations across Australia has significantly increased the number of propagation paths providing suitable sampling of the region. In this work nearly 6500 seismograms recorded in the period from

*Corresponding author: brian.kennett@anu.edu.au

†Present address: ExxonMobil Oil Indonesia Inc., Jakarta, 10210, Indonesia. 
1993-2006 are exploited to provide differential attenuation measurements that are then used to produce seismic attenuation images beneath the Australasian region using a tomographic technique. The resulting images of attenuation structure provide further insights into the lithospheric and asthenospheric structure and temperature distribution beneath the Australian continent and its surroundings.

\section{ATTENUATION STRUCTURE}

The mineral assemblages in the crust and mantle have a very complex rheology; although in the short time span associated with the passage of a seismic wave the behaviour is nearly elastic, some energy is lost through anelastic processes, such as dislocation motion and defect movement. A convenient measure of the rate of energy dissipation is provided by the loss factor $Q^{-1}$, the ratio of the energy loss in a wave cycle to the elastic energy in the oscillation (see e.g. Kennett 2001). An alternative representation of the cumulative attenuation along a propagation path is provided by the quantity $t^{*}=\int \mathrm{d} s Q^{-1} / v$, where the integral is taken along the path with a local propagation wavespeed $v$. We here exploit differential $t^{*}$ between different seismic phases extracted using logarithmic spectral ratios in the frequency domain. We use either $P$ and $S$ phases on the same station, or a set of $P$ or $S$ phases from the same event recorded at multiple stations. Because interpretation of the attenuation results depends on knowledge of the seismic wavespeed we need also to construct a suitable model for the wavespeed distribution.

\section{Seismic data}

Thousands of seismic events from the Australasian region have been archived since 1992 by the Research School of Earth Sciences, Australian National University, from a major program of deployment of portable broadband seismic stations across Australia in experiments primarily designed to improve knowledge of the 3 -D structure of the region. The data coverage exploited in this work is presented in Figure 1, which shows the location of both events and receivers and the great-circle paths between them (in grey). Seismic events with body wave magnitudes between 5.0 and 7.0 with epicentral distances out to $40^{\circ}$ were selected over the

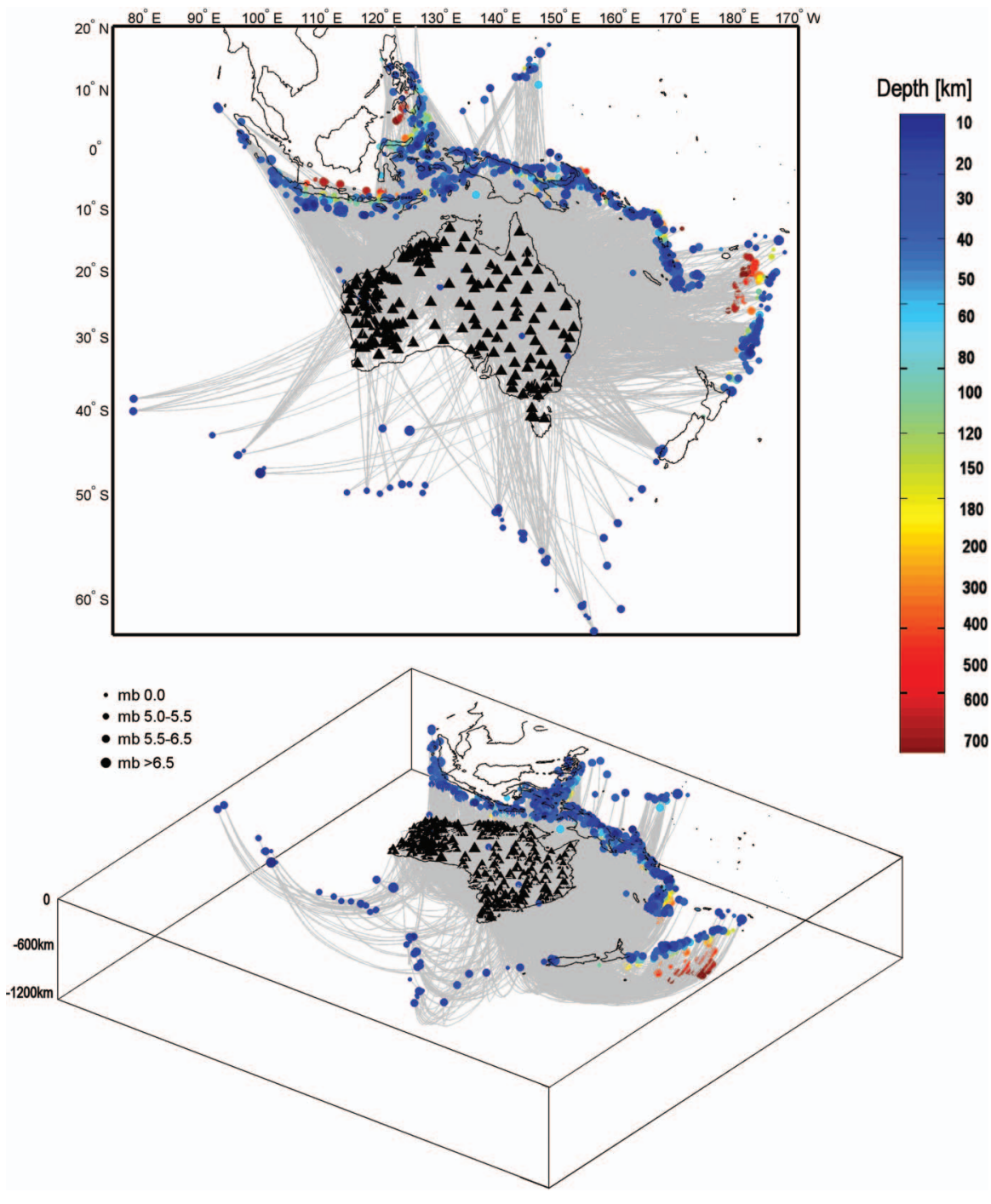

Figure 1 Seismic ray paths exploited in the attenuation study with epicentral distances out to $40^{\circ}$. The great-circle paths from event to receivers are indicated in grey. The portable broadband stations are shown by black triangles. The seismic events are colour coded for depth and the symbol size depends on magnitude $m b$ (between 5.0 and 7.0); $m b$ 0.0 is used where a magnitude is not provided. 
2004. 177. 02. TL13 Time Window: $40 \mathrm{~s}$ Delta: 20.07 [deg] Azim: 341.47 Depth: $70 \mathrm{~km} \quad \mathrm{mb} 6.1$
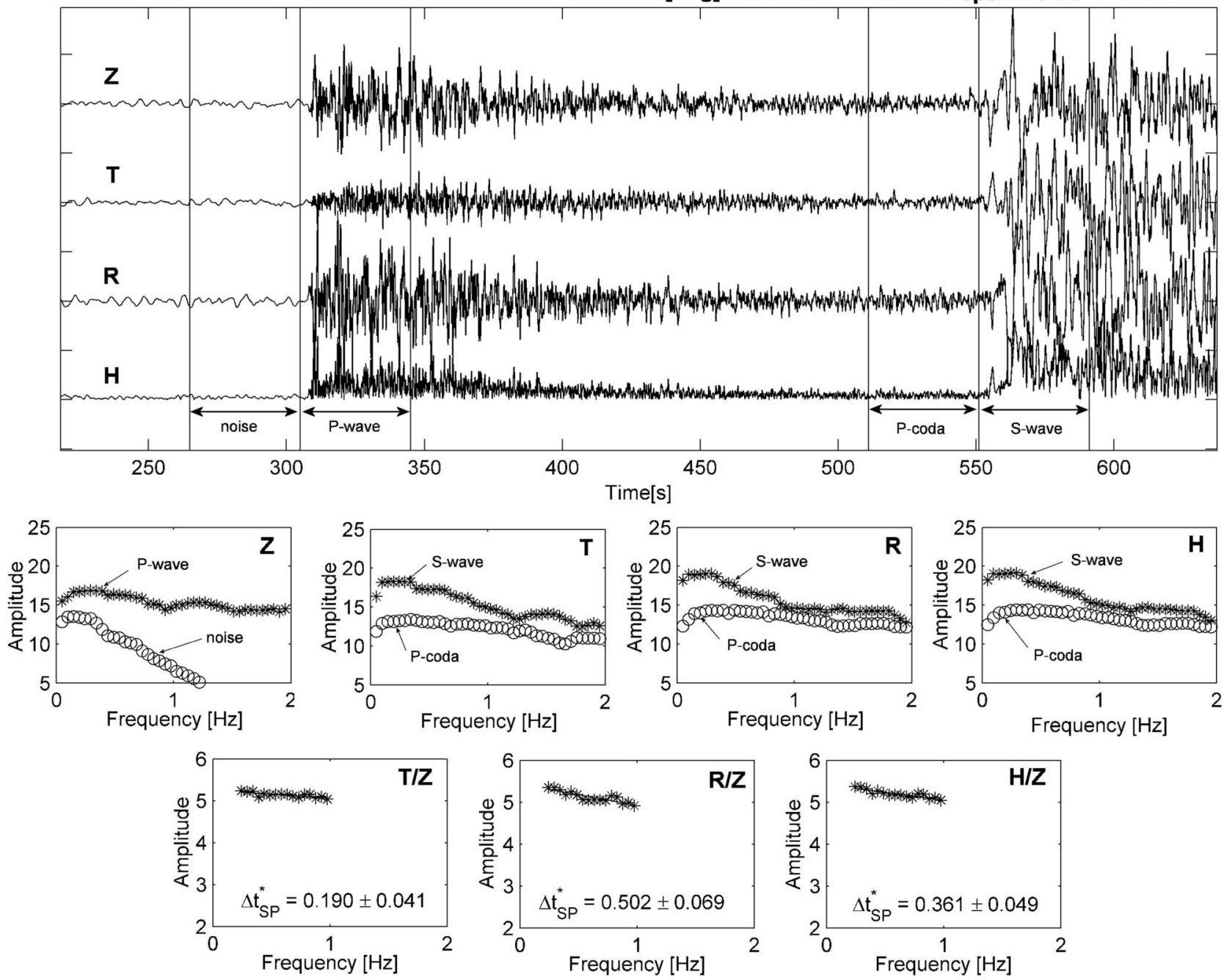

Figure 2 Example of implementation of the spectral ratio method using a graphical user interface (Matlab) for an event near Halmahera at a depth of $70 \mathrm{~km}$ recorded at station TL13. The background noise and the signal spectra for both $P$ and $S$ are estimated using the Multitaper method. The differential attenuation is extracted from the slope of natural logarithm of spectral ratio between $S$ and $P$ spectra over the frequency range from 0.25 to $1.00 \mathrm{~Hz}$. The upper panel displays the seismic traces and the windows used for analysis: $\mathrm{Z}$ vertical component, $\mathrm{R}$, $\mathrm{T}$ radial and transverse components to the path, $\mathrm{H}$ horizontal ground speed. The lower panels show the spectra in each window compared to the preceding noise, and the behaviour of the logarithmic spectral ratios as a function of frequency.

period 1993-2006. The hypocentral parameters were taken from the reprocessed catalogue of Engdahl et al. (1998) and its subsequent updates. The depth range of the events lies between 3 and $670 \mathrm{~km}$, as indicated by the colour coding.

The majority of events in the useful distance range of 5-40 to stations in Australia are located to the north and to the east of Australia in Indonesia, New Guinea, New Caledonia, Tonga to New Zealand, and the Philippines. There are only a limited number of events to the south mainly on the mid-oceanic ridge between Australia and Antarctica; nevertheless, these events still provide valuable information. The northern part of the continent has a good coverage of seismic data except for the Great Sandy Desert area in the northwest, where there are major logistical difficulties for the deployment of portable stations.

\section{Attenuation measurements}

As in the work of Gudmundsson et al. (1994), the differential attenuation is estimated using a spectral ratio method. In the frequency domain at angular frequency $\omega$, the spectrum of a recorded seismic phase for epicentral distance $r$, at an azimuth $\theta$ from the source can be expressed as:

$$
u(\omega, r)=S(\omega) B(\theta) C_{\mathrm{s}}(\omega) M(\omega, r) C_{\mathrm{r}}(\omega, r) I(\omega)
$$

where $S(\omega)$ is the source spectrum, $B(\theta)$ is the source radiation pattern, $C_{s}(\omega)$ is the crustal contribution at the source, $M(\omega, r)$ is the mantle contribution, and $C_{r}(\omega, r)$ is the contribution from the receiver crust. The spectrum imparted by the source is modulated by the instrumental response $I(\omega)$. The main component arising from the mantle 
will be a geometrical spreading factor $g(r)$ and an exponential amplitude loss associated with attenuation:

$$
M(\omega, r)=g(r) \exp \left[-\frac{\omega}{2} \int \frac{\mathrm{d} r}{\mathrm{Q}(\omega, r) v(r)}\right]
$$

For the same event, the spectral ratio between two seismic phases such as $P$ and $S$ cancels most of the effects of the source and instrumental functions. When the phases have similar propagation paths, the crustal effects and radiation pattern also largely cancel. The logarithmic spectral ratio then takes the form:

$$
\begin{aligned}
\ln \frac{\left|u(\omega)_{S}\right|}{\left|u(\omega)_{P}\right|}= & -\frac{\omega}{2}\left[\int_{S} \frac{\mathrm{d} r}{Q_{S} V_{S}}-\int_{P} \frac{\mathrm{d} r}{Q_{P} V_{P}}\right]+\ln g_{S}(r) \\
& -\ln g_{P}(r)+f(\omega, r)
\end{aligned}
$$

where $f(\omega, r)$ is a very weakly varying function of frequency associated with imperfect cancellation of crustal effects in the narrow band of frequencies employed. The geometrical spreading is independent of frequency and so the dominant frequency-dependent contribution is the effect of attenuation. Equation (3) can therefore be expressed as:

$$
\ln \frac{\left|u(\omega)_{S}\right|}{\left|u(\omega)_{P}\right|}=-\frac{\omega}{2}\left(t_{S}^{*}-t_{P}^{*}\right)+c=-\pi f\left(t_{S}^{*}-t_{P}^{*}\right)+c
$$

The logarithmic spectral amplitude ratio thus has a linear dependence on frequency $f$ with a slope determined by the differential attenuation $\Delta t_{S P}^{*}=\left(t_{S}^{*}-t_{P}^{*}\right)$ between the $S$ and $P$ arrivals. This enables estimates of differential attenuation to be extracted by linear regression over the seismic frequency band $(0.25-1 \mathrm{~Hz})$ for which the attenuation is not expected to vary significantly with frequency.

A similar treatment can be made for the same seismic phase from a single event recorded at two different stations to determine differential attenuation factors $\Delta t_{P P}^{*}, \Delta t_{S S}^{*}$. When an event is recorded by a number of stations it is convenient to designate one as a reference and carry out the differential attenuation measurements with respect to that station.

Differential attenuation measurements have been made from nearly 6500 high-quality three component seismograms using a graphical user interface implemented in the Matlab computer programming environment (Figure 2). Spectra for the $P$ phase are extracted from the vertical component $(Z)$ and $S$ phase spectra from the transverse $(T)$ and radial component $(R)$, after rotation to the great-circle between source and receiver. In addition, the horizontal ground speed $H$, $\sqrt{ }\left(R^{2}+T^{2}\right)$, is constructed from the $R$ and $T$ traces; the quantity $H$ has the advantage of not depending on the orientation of the path. The spectra are calculated for a time window of between 25 and $45 \mathrm{~s}$ and compared with the noise in the immediately preceding window. The location of the phase windows are predicted using the ak135 model of Kennett et al. (1995), and then adjusted by hand picking to obtain a more accurate estimate. The spectral windows start $1 \mathrm{~s}$ before the estimated $\mathrm{P}$ arrival time and $3 \mathrm{~s}$ before the $\mathrm{S}$ pick. The travel times for the $\mathrm{P}$ and $S$ phases are used to build the 3-D velocity structure necessary to interpret the differential attenuation measurements.

Figure 2 illustrates the measurement of the differential attenuation between $S$ and $P$ waves $\left(\Delta t_{S P}^{*}\right)$ for an event near Halmahera at a depth of $70 \mathrm{~km}$. The station lies at an epicentral distance of $20^{\circ}$ from the station TL13 in southern Australia. No filtering has been applied to the velocity seismograms. The time windows used to estimate the spectra for signal and background noise for both $\mathrm{P}$ and $\mathrm{S}$ waves are $40 \mathrm{~s}$ long. All the spectra are estimated using the multitaper method (Percival \& Walden 1993), which provides a stable spectral estimate. As would be expected the spectrum for the $S$ phase spectrum decays more rapidly than that for the $P$ phase, but both show a similar character with frequency. The

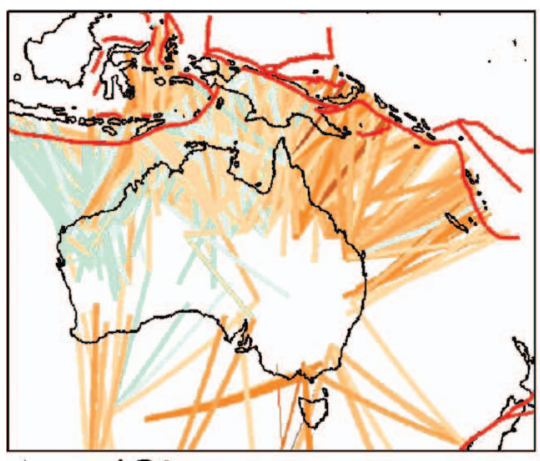

$\Delta:<18^{\circ}$

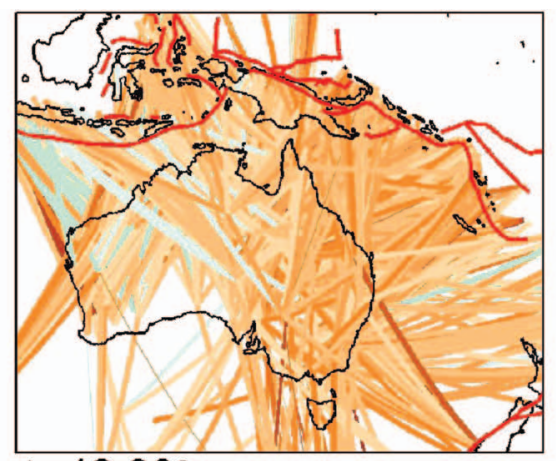

$\Delta: 18-30^{\circ}$

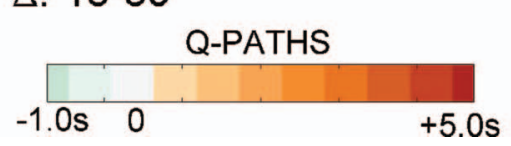

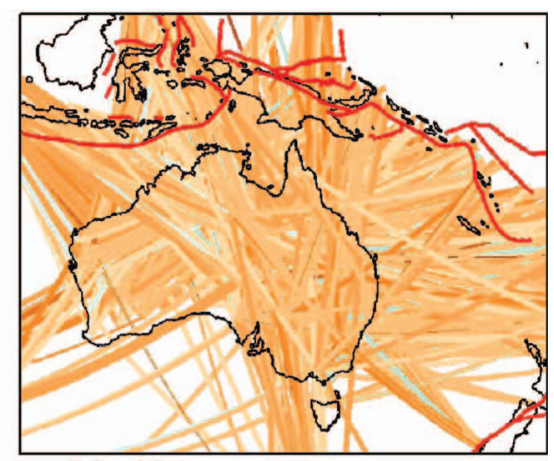

$\Delta: 28-40^{\circ}$ 
differential attenuation $\left(\Delta t_{S P}^{*}\right)$ is extracted from the slope of the natural logarithm of the spectral ratio between the $S$ and $P$ phases over the restricted frequency range from 0.25 to $1.00 \mathrm{~Hz}$.
Figure 3 provides a summary of the $\Delta t^{*}{ }_{S P}$ differential attenuation results for the Australian seismic dataset. The great-circle paths between source and receiver are plotted and colour coded by the value of $\Delta t^{*}{ }_{S P}$ for three


S wave perturbation (\%) relative to ak135

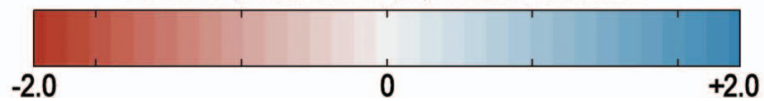

Figure 4 Chequer-board test of the recovery of velocity structure from tomographic inversion at 35-120, 120-220 and 220-320 km depth with different cell sizes: (ad) $9^{\circ}$ and $(\mathrm{e}-\mathrm{h}) 6^{\circ}$. The panels $(\mathrm{b}-\mathrm{d})$ and $(\mathrm{f}-\mathrm{h})$ represent the recovered images for each tomographic inversion. (a) and (e) are the input structure where the anomalies have maximal perturbations of $\pm 2 \%$ relative to the $a k 135$ model. 
groups of epicentral distances $\Delta: 5-18^{\circ}$ sampling the lithosphere, $18-30^{\circ}$ with waves returned from the transition zone in the upper mantle, and $28-40^{\circ}$ with arrivals turning below the $660 \mathrm{~km}$ discontinuity. The $\Delta t_{S P}^{*}$ values represent the average for the path between source and receiver. At first sight negative values for $\Delta t^{*}{ }_{S P}$ may seem surprising, but the variation in the $v_{p} / v_{s}$ ratio with depth mean that $\mathrm{P}$ and $\mathrm{S}$ waves do not have the same propagation paths. It is thus possible for $\mathrm{P}$ waves to sample the lower $Q$ asthenosphere while $S$ waves remain within the higher $Q$ lithosphere with less loss. This feature is seen in the cratonic areas of northern and western Australia that have a relatively thick lithosphere.

\section{Tomographic inversion}

In a similar way to the travel times of $\mathrm{P}$ and $\mathrm{S}$ waves, the differential attenuation measurements for $\Delta t_{S P}^{*}$ represent an integral along the propagation path. For the $\mathrm{P}$ and $\mathrm{S}$ phases, the differential measurements $\Delta t_{P P}^{*}$ and $\Delta t_{S S}^{*}$ represent the difference of two path integrals. We are therefore able to employ the attenuation results in a two-stage tomographic inversion based on the treatment of Rawlinson \& Sambridge (2003). First, we exploit the arrival times of the $P$ and $S$ wave arrivals for all the paths illustrated in Figure 1 to develop a 3-D model for the seismic wavespeeds in the upper mantle. Then, we are able to invert for the 3-D distribution of the loss factor $Q^{-1}$, by calculating the requisite path integrals in a proposed model and then comparing the results to the observations.

We employ a cellular model to represent both the seismic wavespeed and the attenuation structure, with uniform properties in each cell. To ensure stability in the tomographic inversion we employ regularisation through a combination of damping towards a background model and constraints on gradients to produce a smoothly varying model. The station distribution across the continent has some holes and the regularisation provides a means of interpolation across such data gaps.

The model volume encompasses the region of interest around Australia: from latitude $22^{\circ} \mathrm{N}$ to $65^{\circ} \mathrm{S}$, longitude $78^{\circ}$ to $189^{\circ} \mathrm{E}$ and from 0 to $1240 \mathrm{~km}$ in depth. The lithosphere and the mantle are divided into 10 layers with layer thicknesses ranging from 35 to $100 \mathrm{~km}$ in the lithosphere and upper mantle and from 100 to $200 \mathrm{~km}$ in the transition zone and upper mantle. We use a uniform grid of constant velocity and attenuation cells with horizontal size $3^{\circ} \times 3^{\circ}$. Several cell sizes were examined $\left(1^{\circ} \times 1^{\circ}, 2^{\circ} \times 2^{\circ}\right.$ and $\left.3^{\circ} \times 3^{\circ}\right)$, and we found that the best balance between resolution and ray sampling was

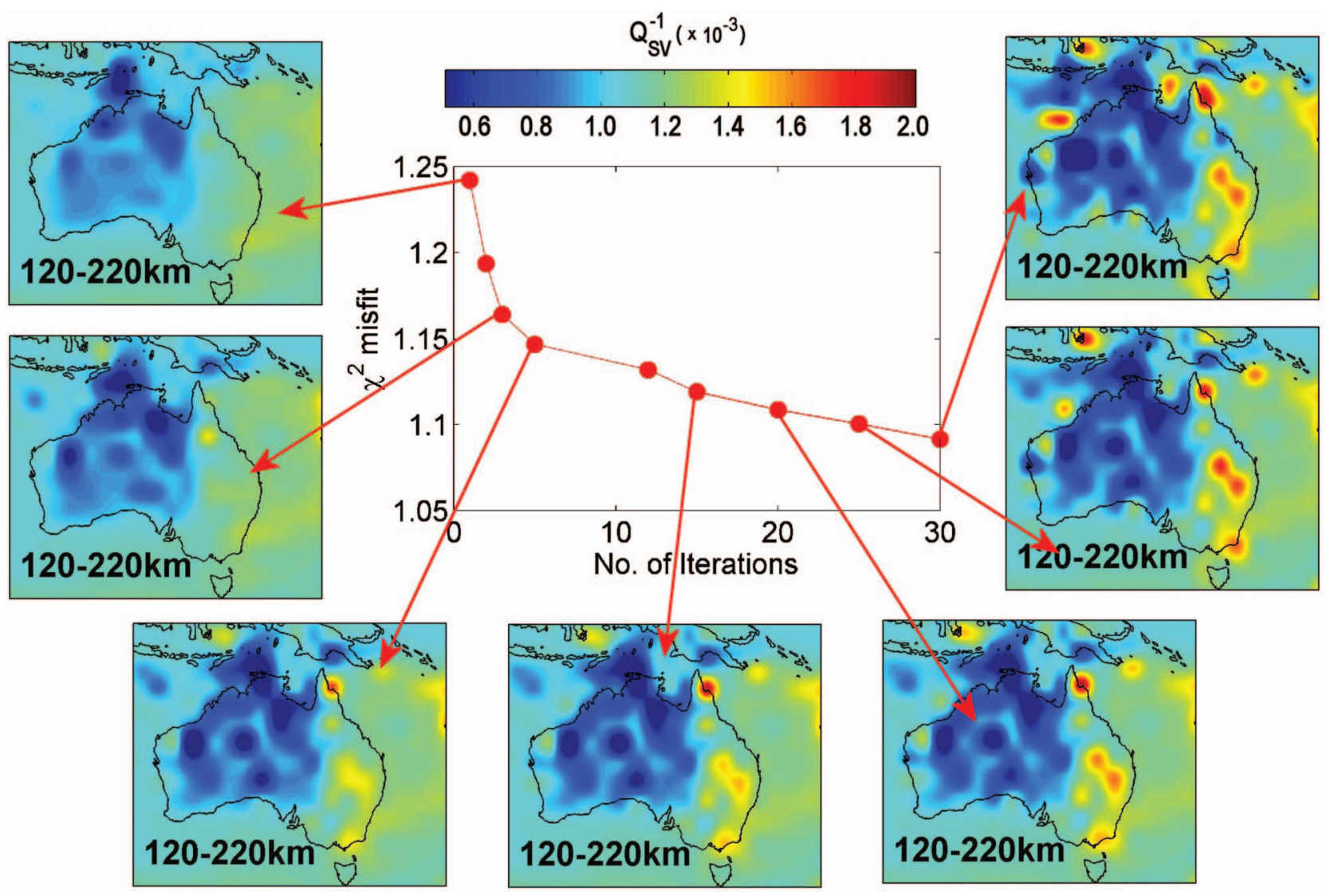

Figure 5 Misfit function $\left(\chi^{2}\right)$ for the inversion, for attenuation from $\Delta t_{S P}^{*}$, as a function of the number of iterations, with the corresponding attenuation images for the depth interval from 120-220 km. As the number of iterations increases, smaller scale features become prominent. 
achieved with $3^{\circ} \times 3^{\circ}$ cells. With this model parameterisation, the total number of unknowns to be extracted from the tomographic inversion is about 11,100 .

\section{Chequer-board tests}

Before producing tomographic images from the inversion of the Australian data set, a set of chequer-board tests were carried out to evaluate the model parameterisations and the inversion method. We examine how well artificial wave-speed anomalies are recovered upon inversion using the actual geometry of sources and receivers. The 3-D ray-tracing is undertaken using the fast-marching method (Rawlinson \& Sambridge 2003) that provides a stable means of tracking wavefronts through complex structures (Rawlinson \& Sambridge 2004).

In this chequer-board test, a set of synthetic models are divided into alternating regions of high and low wavespeed with patch sizes of $9^{\circ} \times 9^{\circ}$ or $6^{\circ} \times 6^{\circ}$. The input model is a simple structure of $S$ wave perturbation with the anomalies having maximum perturbations of $\pm 2 \%$ relative to the ak135 model (Kennett et al. 1995) as illustrated in Figure $4 \mathrm{a}$, e. Figures $4 \mathrm{~b}-\mathrm{d}$ and $4 \mathrm{f}-\mathrm{h}$ show the results from the inversions for these imposed structures for particular depth slices. The quality of the inversion can be judged by the extent of the recovery of the original chequer-board pattern. The recovery of structure is quite good in the northern part of Australia where the raypath density is high. Although the southern margin of the continent has limited ray sampling, the chequer-board tests still show a reasonable result. Parts of the structure show some degree of streaking associated with the dominant directions of wave propagation. As is inevitable with limited sampling there is some loss in the amplitude of the patterns recovered in the tomographic inversion.

\section{Attenuation models}

The initial model used for 3-D ray tracing was derived from the shear wavespeed results of Fishwick et al. (2005) from surface wave tomography. The ray tracing was carried out using the fast-marching method of Rawlinson \& Sambridge (2003). Wavespeed inversion is then applied to determine the deviations from the initial model, and then the resulting velocity field is used in the inversion of the differential attenuation results $\left(\Delta t_{S P}^{*}, \Delta t_{P P}^{*}\right.$ and $\left.\Delta t_{S S}^{*}\right)$ to produce shear (transverse and radial) and compressional attenuation models
Figure 6 Transverse and radial shear attenuation $\left(Q_{S H-S P}^{-1}\right.$ and $Q_{S H-S P}^{-1}$ ) beneath the Australasian region in the depth ranges $35-120$, $120-220$ and $220-320 \mathrm{~km}$. Note that the colour scales employed for $Q^{-1}$ in the lithosphere and the upper mantle are different.
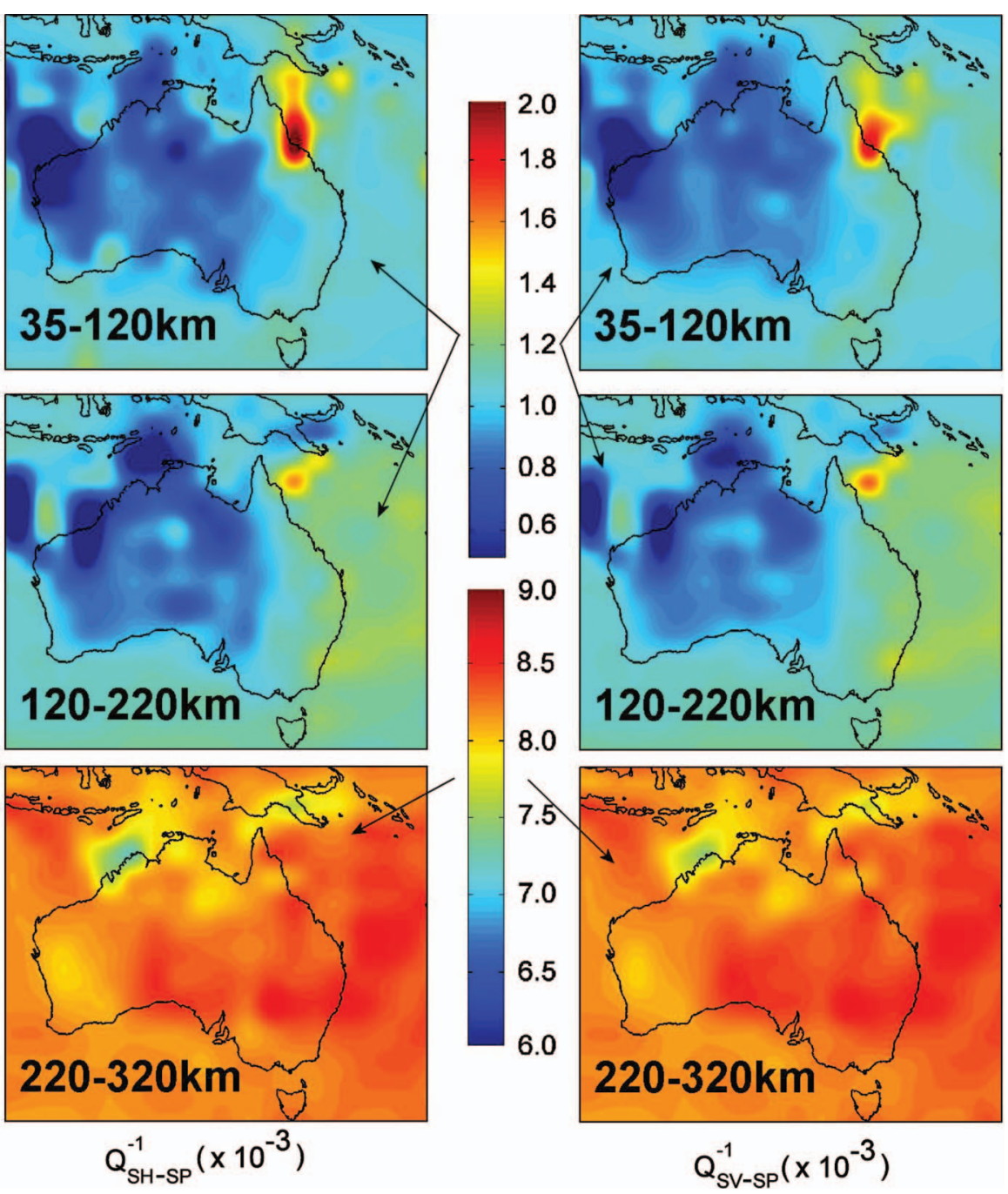
$\left(Q_{S H}^{-1}, Q_{S V}^{-1}\right.$ and $\left.Q_{P}^{-1}\right)$. The inversions employ stronger damping than smoothing constraints. The wavespeed inversions generate structures that are compatible with previous studies, but which are tuned to the specific needs of the attenuation inversion with direct sampling of the paths used in the attenuation measurements.

To distinguish the attenuation models produced from the various inversions using the $\Delta t_{S P}^{*}, \Delta t_{P P}^{*}$ and $\Delta t_{S S}^{*}$ information, we employ the following notation. Differential attenuation from the $\Delta t_{S P}^{*}$ results is designated $Q_{S H-S P}^{-1}$ and $Q_{S V-S P}^{-1}$ depending on the shear component employed; transverse $(S H)$ and radial $(S V)$. Similarly, we use $Q_{S H-S S}^{-1}$ and $Q_{S V-S S}^{-1}$ for the transverse and radial shear attenuation obtained from the $\Delta t_{S S}^{*}$ results and $Q_{P-P P}^{-1}$ for compressional attenuation from the $\Delta t_{P P}^{*}$ results.

Since the kernel for the inversion is quite sensitive to the number of iterations, data and model covariance, the misfit $\chi^{2}$ is used as our quality control. A cross plot of the misfit versus number of iterations is shown in Figure 5 accompanied by the corresponding tomographic images for the depth interval $120-220 \mathrm{~km}$. The misfit parameter lies between 1.05 to 1.25 for iterations $1-30$. When the misfit value is relatively small there is no significant difference between observed data and its model. The value of the misfit reduces as the number of iterations increases, but also finer scale features also emerge in the images. We stop the iterations once we have both a good level of fit (i.e. the misfit function start to converge) and reasonable geological features. In this set of inversions we stop at the fifth iteration, at which point the main structure has appeared.

The 3-D models for $Q_{S H-S P}^{-1}$ and $Q_{S V-S P}^{-1}$ beneath the Australasian region are displayed in Figure 6 for the depth intervals $35-120,120-220$ and $220-320 \mathrm{~km}$. In order to present the variations in the much higher attenuation in the upper mantle $(220-320 \mathrm{~km})$, the $Q^{-1}$ images at this level are plotted with different colour scale to that used for the lithosphere.

The results show a distinctive pattern of attenuation, consistent with the observations in Figure 3, with a relatively low attenuation in the lithosphere in the centre and west of Australia compared with the eastern part. The fast seismic lithosphere extends to $220 \mathrm{~km}$ in the west and so is well captured by the simple parametrisation in depth. In the east beneath the Paleozoic fold belts the lithosphere is of the order of $120 \mathrm{~km}$ thick (Fishwick et al. 2008) and so there will be asthenospheric influences in the images for the layer from $120-220 \mathrm{~km}$. There is little difference between the attenuation structures estimated using information
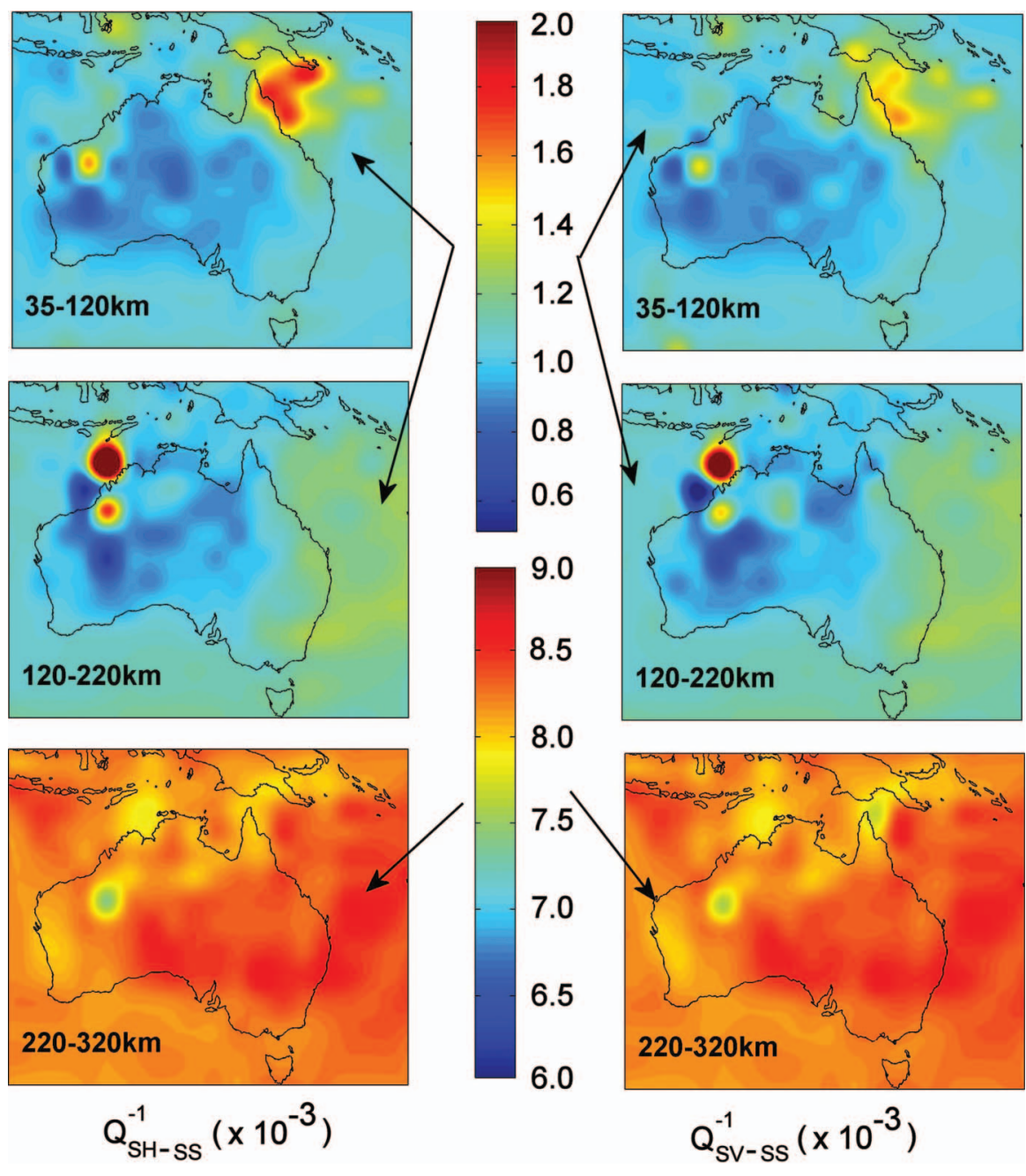

Figure 7 Transverse and radial shear attenuation $\left(Q_{S H-S S}^{-1}\right.$ and $Q_{S H-S S}^{-1}$ ) beneath Australasian region in the depth ranges $35-120$, 120-220 and $220-320 \mathrm{~km}$ derived from inter-station differential $Q_{S H-S S}^{-1}$ measurements. Note that the colour scales employed for $Q^{-1}$ in the lithosphere and the upper mantle differ. 
from the radial and transverse components, even though surface wave studies suggest significant polarisation anisotropy for wavespeeds (Debayle \& Kennett 2001; Fichtner et al. 2010). A necessary consequence of transverse anisotropy in shear wavespeed is that the attenuation should also be anisotropic, but such an effect does not need to be large.

Both the $Q_{S H-S P}^{-1}$ and $Q_{S V-S P}^{-1}$ images for the upper mantle (220-320 km) suggest very high attenuation $\left(Q^{-1}\right.$ between 0.0072 and $0.0085 ; Q_{S-S P} \approx 120-140$ ) compared with the lithosphere above. In the northern part of Australia, where the path density is higher, the results suggest that there is lower attenuation than in the southern part.

The separate inversions for $Q_{S H-S S}^{-1}$ and $Q_{S V-S S}^{-1}$ images give independent verification for the determination of lithospheric and upper mantle attenuation features beneath the Australasian region (Figure 7). Further, the $Q_{S H-S S}^{-1}$ and $Q_{S V-S S}^{-1}$ images show similarities and consistency with the $Q_{S H-S P}^{-1}$ and $Q_{S V-S P}^{-1}$ images. The consistent features are the low loss lithosphere beneath central-western Australia, high attenuation beneath eastern sea board, and very high attenuation around Coral Sea and very high attenuation with low variation in the upper mantle.

The loss factors $Q_{S H-S S}^{-1}$ and $Q_{S V-S S}^{-1}$ for the lithosphere beneath the central-western region of Australia lie between 0.0007 and $0.0009\left(Q_{S-S S} \approx 1100-1450\right)$. Beneath the eastern seaboard there is somewhat greater loss with $Q^{-1}$ between 0.0012 and $0.0014\left(Q_{S-S S} \approx 715-\right.$ 833). The $\Delta t_{P P}^{*}$ data are inverted to yield determination of compressional attenuation structure beneath Australasian region. Compared with the $\Delta t_{S S}^{*}$, the $\Delta t_{P P}^{*}$ data are relatively free of noise; so that the inverted $Q_{P-P P}^{-1}$ structure should be more robust than for $Q_{S-S S}^{-1}$. Despite this limitation, the $Q_{S H-S S}^{-1}$ and $Q_{S V-S S}^{-1}$ patterns still show strong similarities with those constructed for $Q_{P-P P}^{-1}$.

Figure 8 shows the $Q_{P-P P}^{-1}$ images of the Australasian region in the depth intervals 35-120, 120-220 and 220 $320 \mathrm{~km}$. Note that the scale employed for the $\mathrm{P}$ wave attenuation $Q_{P-P P}^{-1}$ is half that used for $Q_{S-S S}^{-1}$ in Figure 7 . The results once again show strong similarities with the attenuation images for $S$ waves. In the lithosphere beneath central-western Australia, the values of $Q_{P-P P}^{-1}$ lie between 0.0003 and $0.0005\left(Q_{P-P P} \approx 2000-3300\right)$; energy loss in propagation is minimal and high frequency waves propagate to considerable distance (Kennett \& Furumura 2008).

Beneath the eastern seaboard $Q_{P-P P}^{-1}$ has values between 0.0005 and $0.0006\left(Q_{P-P P} \approx 1700-2000\right)$. As seen in the other attenuation images, the attenuation in the shallow structure around the Coral Sea is somewhat higher than its surroundings reaching $0.0007\left(Q_{P-P P} \approx\right.$ 1400). In the upper mantle the values of $Q_{P-P P}^{-1}$ lie between 0.004 and $0.0043\left(Q_{P-P P} \approx 230-250\right)$.

\section{DISCUSSION}

The tomographic images of attenuation structure (Figures 6-8) provide clear evidence for variations in seismic attenuation beneath the Australasian region. The regions beneath the western-central Australian cratons show very little seismic attenuation (see e.g. Kennett \& Furumura 2008), but there is a sharp change in depth, since those $S$ waves that penetrate into the asthenosphere rapidly lose high-frequency energy (Gudmundsson et al. 1994). In the east of Australia, lower seismic wavespeeds are correlated with an increase in seismic attenuation.

Figure 9 shows a comparison between seismic wavespeed and seismic attenuation images for the lithosphere beneath the Australasian region. The seismic wavespeed image is derived from the wavespeed model of Fishwick et al. (2005) constructed from surface wave tomography. The $Q_{P}^{-1}$ model is constructed from

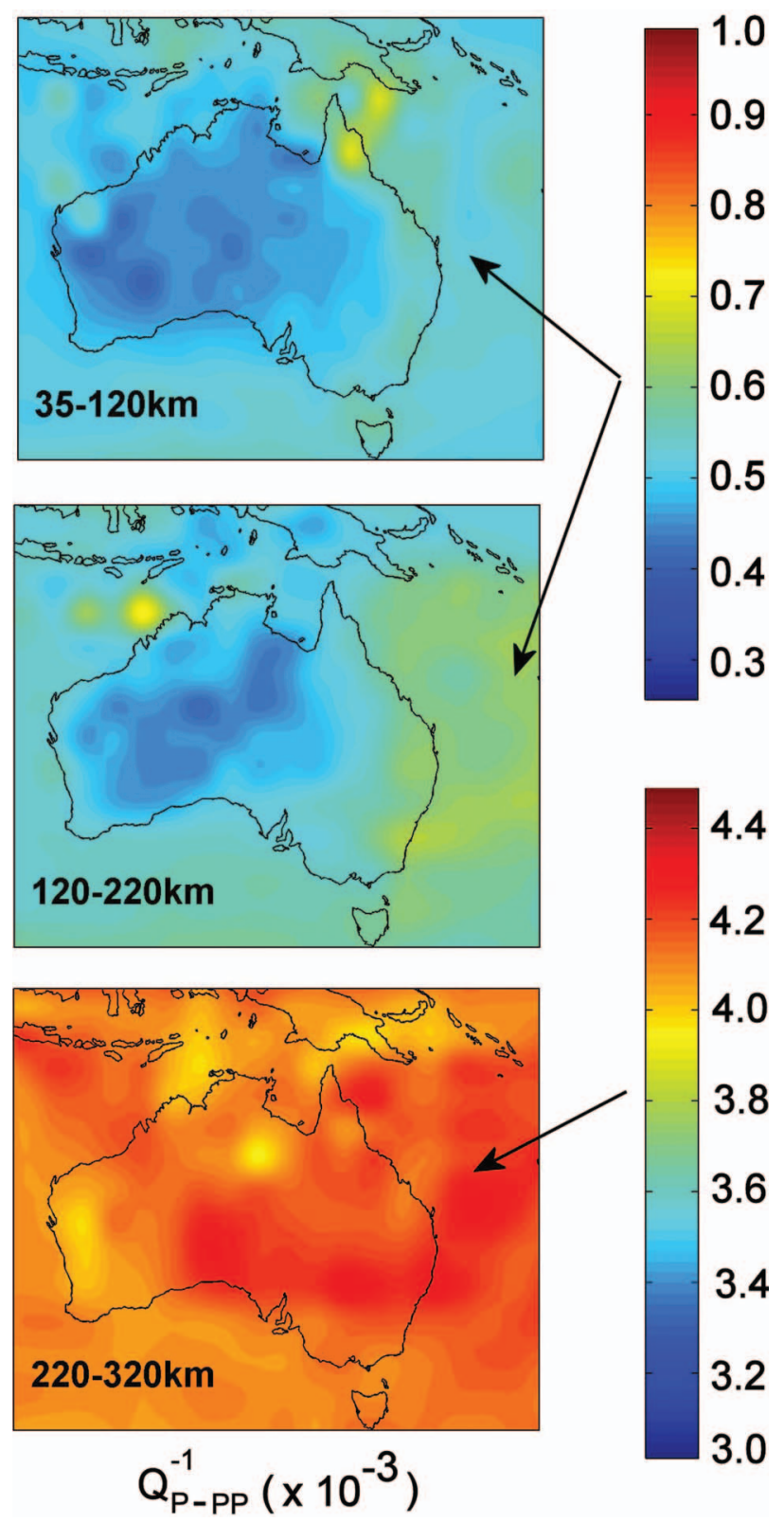

Figure 8 Compressional attenuation $\left(Q_{P-P P}^{-1}\right)$ beneath Australasian region in the depth ranges $35-120,120-220$ and 220 $320 \mathrm{~km}$ derived from inter-station differential $Q_{P-P P}^{-1}$ estimates. Note that once again the colour scales employed for $Q^{-1}$ in the lithosphere and the upper mantle differ. 


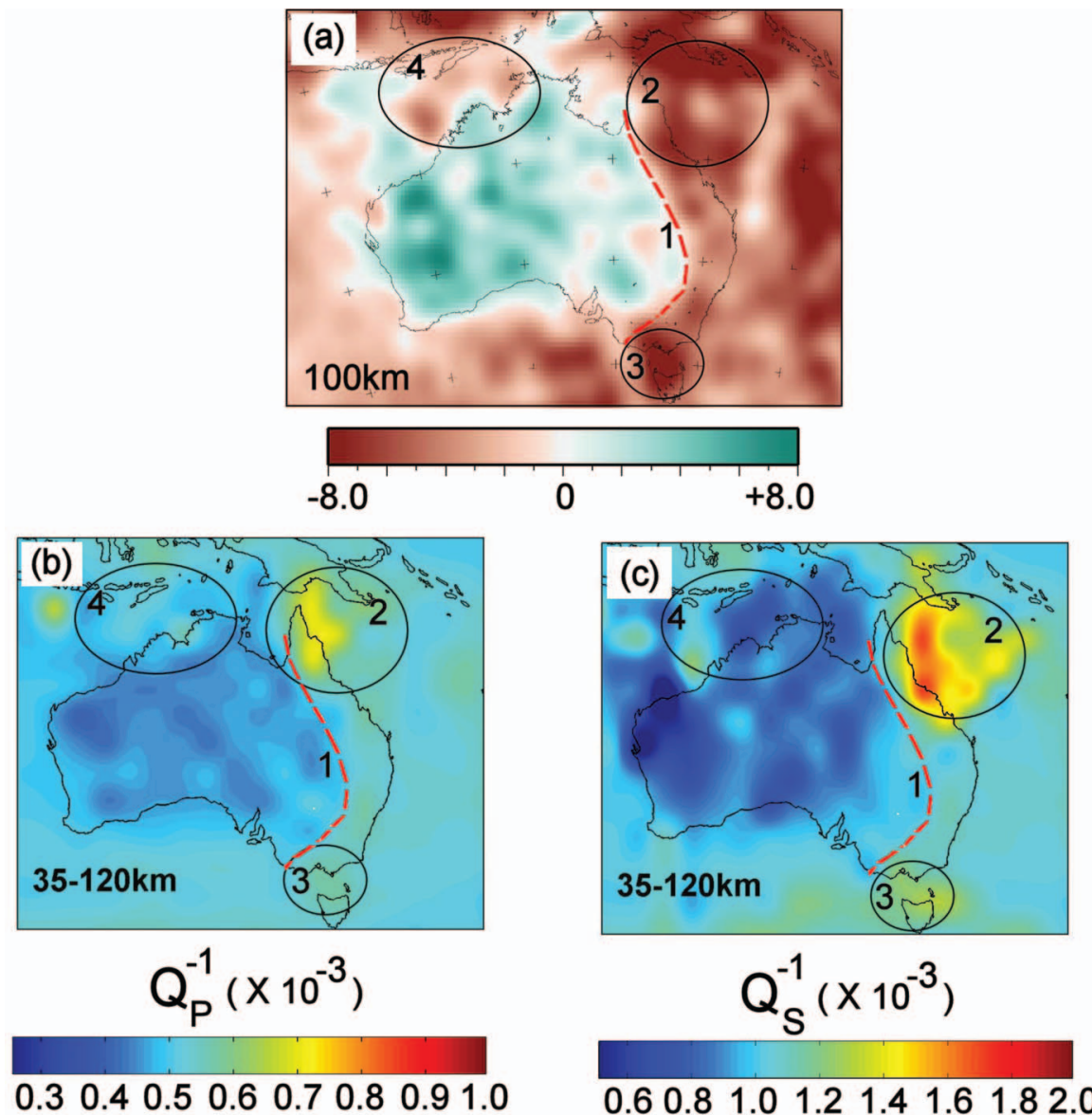

Figure 9 Comparison between (a) $S$ wavespeed images derived from surface wave tomography (model from Fishwick et al. 2005), (b) compressional wave attenuation $Q_{P}^{-1}$ and (c) shear wave attenuation $Q_{P}^{-1}$.

the differential attenuation measurements for $\mathrm{P}$ and the $Q_{S}^{-1}$ model by combining the full set of results for $S$ attenuation. We see that in the lithosphere beneath the Australasian region, the values of $Q_{P}^{-1}$ and $Q_{S}^{-1}$ are generally inversely correlated with the variations in shear wavespeed. Figure 9 shows that the abrupt change from high shear wavespeed to low wavespeed for anomaly (1) is also marked as a sharp change from low to high $Q_{P}^{-1}$ and $Q_{S}^{-1}$ (approximately in the location of the controversial Tasman Line marking the eastern edge of Precambrian material). A similar correlation also exists in several other areas: (2) low seismic S wavespeed at around $100 \mathrm{~km}$ beneath the Coral Sea is correlated with significantly increased $Q_{P}^{-1}$ and $Q_{S}^{-1}$; (3) low seismic wavespeed around Bass Strait is associated with high attenuation. To the north of Australia, (4) low seismic $S$ wavespeed is well correlated with $Q_{P}^{-1}$ and fairly well correlated with $Q_{S}^{-1}$.

The existence of hot spots beneath the Coral Sea and Bass Strait is well marked by localised high attenuation, most likely because seismic attenuation has a stronger sensitivity to temperature than seismic wavespeed.

\section{CONCLUSIONS}

The seismic attenuation below the Australian continent shows considerable variability. A low loss lithosphere extends to at least $200 \mathrm{~km}$ depth across the cratonic zones of central and western Australia. Corrobative evidence for this region of low seismic attenuation comes from observations of high frequency $\mathrm{P}$ and $\mathrm{S}$ waves from subduction zone events to $18^{\circ}$ from the source, with very little difference in the frequency content of $\mathrm{P}$ and $\mathrm{S}$. This requires very high $Q$, with $Q_{S}$ certainly greater than 1000 .

Along the eastern margin the attenuation increases below $120 \mathrm{~km}$ depth, which is consistent with the surface wave study of Dalton et al. (2008) that indicates high attenuation rising to about $150 \mathrm{~km}$ along the eastern coastline. The mixture of high and low loss material in our coarse $3^{\circ} \times 3^{\circ} \times 100 \mathrm{~km}$ cells tends to mute this influence.

Beneath the low loss lithosphere, the asthenosphere shows much higher attenuation with reductions in $\mathrm{Q}$ by a factor of $6-10$. The relatively coarse parameterisation that is compatible with our data constraints probably exaggerates the nature of the jump, but a strong contrast is required.

Experimental work on the properties of upper-mantle materials at seismic frequencies (Jackson 2000) shows that the influence of temperature on attenuation becomes apparent well before the solidus is reach and before the seismic wavespeeds are significantly affected. Variations in temperature are therefore expected to play a large part in controlling attenuation behaviour. This sensitivity can be used to correlate seismic attenuation anomalies in the mantle with temperature variations (see e.g. Mitchell 1995; Romanowicz 1995). 
Goes et al. (2005) have attempted to extract estimates of temperature from the shear wavespeed models of Yoshizawa \& Kennett (2004) and Simons et al. (2002). The model for the variation of seismic wavespeed with temperature uses both elastic and anelastic effects in a third-order finite strain representation, coupled to a conductive and steady-state model of heat transport. The $\pm 8 \%$ variations in seismic wavespeed yield a wide range of temperatures that correlate well between the two seismic velocity models. There is a strong correlation between the temperature estimates by Goes et al. (2005) and the attenuation variations with the lithosphere beneath Australia. For instance, in central-western Australia, the boundary of the low attenuation anomaly with $Q_{S}^{-1}<0.0008\left(Q_{S}>1250\right)$ corresponds approximately to the $1000^{\circ} \mathrm{C}$ isotherm from Goes et al. (2005). The higher attenuation $Q_{S}^{-1}=0.0013\left(Q_{S} \approx 770\right)$ in the eastern part of Australia is similarly associated with temperature estimates around $1400^{\circ} \mathrm{C}$. The high attenuation anomalies along eastern coast of Australia correlate with the presence of recent volcanism in northern Queensland and the Newer Volcanic Province in Victoria.

\section{ACKNOWLEDGEMENTS}

This work has been supported through an ANU PhD Scholarship to Agus Abdullah and Australian Research Council Discovery Grants. Thanks are extended to many members of the Seismology Group at the Research School of Earth Sciences, Australian National University, for their support in the field and in data handling.

\section{REFERENCES}

CHENG H-X. 2000. Seismic body wave attenuation in the upper mantle beneath the Australian continent. PhD thesis, Research School of Earth Sciences, The Australian National University.

Dalton C. A., Ekstrom G. \& Dziewonski A. M. 2008. The global attenuation structure of the upper mantle, Journal of Geophysical Research 113, B09303, doi:10.10292007JB005429.

Debayle E. \& KenNetT B. L. N. 2001. Anisotropy in the Australian upper mantle from Love and Rayleigh wave inversion. Earth and Planetary Science Letters 184, 339-351.

ENGDAHL E. R., VAN DER HILST R. D. \& BULAND R. 1998. Global teleseismic earthquake relocation with improved travel times and procedures for depth determination. Bulletin of the Seismological Society of America 88, 722-743.

Fichtner A., KennetT B. L. N., Igel H. \& Bunge HP-. 2010. Full seismic waveform tomography for radially anisotropic structure: New insights into the past and present states of the Australasian upper mantle. Earth and Planetary Science Letters 290, 270-280.
Fishwick S., KennetT B. L. N. \& ReAding A. M. 2005. Contrasts in lithospheric structure within the Australian Craton. Earth and Planetary Science Letters 231, 163-176.

Fishwick S., Heintz M., KennetT B. L. N., ReAding A. M. \& YoshIZAWA K. 2008. Steps in lithospheric thickness within eastern Australia, evidence from surface wave tomography. Tectonics 27, TC0049, doi:10.129/2007TC002116.

Goes S., Simons F. J. \& YoshizAWA K. 2005. Seismic constraints on temperature of the Australian uppermost mantle. Earth and Planetary Science Letters 236, 227-237.

Gudmundsson O., KennetT B. L. N. \& Goody A. 1994. Broadband observations of upper mantle seismic phases in northern Australia and the attenuation structure in the upper mantle. Physics of the Earth and Planetary Interiors 84, 207-226.

JACKSON I. 2000. Laboratory measurements of seismic wave disper sion and attenuation: recent progress. In: Karato S., Forte A. M. Liebermann R. C., Masters G. \& Stixrude L. eds. Earth's Deep interior: mineral physics and tomography from the atomic to the global scale. AGU Geophysical Monograph 117, 265-289.

KENNETT B. L. N. 2001. The seismic wavefield, I: introduction and theoretical development. Cambridge University Press, Cambridge, $\mathrm{xii}+370 \mathrm{pp}$.

KenNetT B. L. N. 2003. Seismic structure in the mantle beneath Australia. In: Hillis R. R \& Müller R. D. eds. Evolution and dynamics of the Australian Plate. pp. 7-23. Geological Society of Australia Special Publication 22 and Geological Society of America Special Paper 372

KenNetT B. L. N. \& FurumurA T. 2008. Stochastic waveguide in the Lithosphere: Indonesian subduction zone to Australian Craton. Geophysical Journal International 172, 363-382.

KennetT B. L. N., Engdahl E. R. \& Buland R. 1995. Constraints on seismic velocities in the earth from travel times. Geophysical Journal International 122, 108-124.

MitChelL B. J. 1995. Anelastic structure and evolution of the continental crust and upper mantle from seismic surface wave attenuation. Reviews of Geophysics 33, 441-462.

PERCIVAL D. B. \& WALDEN A. T. 1993. Spectral analysis for physical applications: multitaper and conventional univariate techniques. Cambridge University Press, Cambridge.

RAWLINSON N. \& SAMBRIDGE M. 2003. Seismic traveltime tomography of the crust and lithosphere. Advances in Geophysics 46, 81-198.

RAWLINSON N. \& SAMBRIDGE M. 2004. Wavefront evolution in strongly heterogeneous layered media using the fast marching method. Geophysical Journal International 156, 631-647.

RomANOwicz B. 1995. A global tomographic model of shear attenuation in the upper mantle. Journal of Geophysical Research $\mathbf{1 0 0}$, $12375-12394$

SAMBRIDGE M. 1999. Geophysical inversion with a neighbourhood algorithm-I. Searching a parameter space. Geophysical Journal International 138, 479-494.

Simons F. J., van DeR Hilst R. D., Montagner J. P. \& Zielhuis A 2002. Multimode Rayleigh wave inversion for heterogeneity and azimuthal anisotropy of the Australian upper mantle. Geophysical Journal International 151, 738-754.

YoshizAWA K. \& KenNETT B. L. N. 2004. Multimode surface wave tomography for the Australian region using a three-stage approach incorporating finite frequency effects. Journal of Geophysical Research 109, 1-19.

Received 26 October 2010; accepted 19 December 2010 